\title{
Simulated reconstruction of the remote dipole field using the kinetic Sunyaev Zel'dovich effect
}

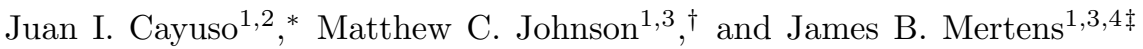 \\ ${ }^{1}$ Perimeter Institute for Theoretical Physics, Waterloo, Ontario N2L 2Y5, Canada \\ ${ }^{2}$ Department of Physics and Astronomy, University of Waterloo, Waterloo, ON, N2L 3G1, Canada \\ ${ }^{3}$ Department of Physics and Astronomy, York University, Toronto, Ontario, M3J 1P3, Canada and \\ ${ }^{4}$ Canadian Institute for Theoretical Astrophysics, \\ University of Toronto, Toronto, ON M5H 3 H8 Canada
}

\begin{abstract}
The kinetic Sunyaev Zel'dovich (kSZ) effect, cosmic microwave background (CMB) anisotropies induced by scattering from free electrons in bulk motion, is a primary target of future CMB experiments. In addition to shedding light on the distribution of baryons and the details of the epoch of reionization, measurements of the $\mathrm{kSZ}$ effect have the potential to address fundamental questions about the structure and evolution of our Universe on the largest scales and at the earliest times. This potential is unlocked by combining measurements of small-scale CMB anisotropies with large-scale structure surveys, a technique known as kSZ tomography. Previous work established a quadratic estimator for the remote dipole field, the CMB dipole observed at different locations in the Universe, given a CMB map and a redshift-binned map of large scale structure. This previous work did not include gravitational lensing, redshift space distortions, or non-linear evolution of structure. In this paper, we investigate how well the remote dipole field can be reconstructed in the presence of such effects by using mock data from a suite of simulations of gigaparsec-sized regions of the Universe. To properly model both large and small scales, we develop a novel box-in-box simulation pipeline, where small-scale information is obtained from L-PICOLA N-body simulations, and large-scale information obtained by evolving fields using linear theory and adding the resulting corrections to the N-body particle data. This pipeline allows us to create properly correlated maps of the primary CMB including lensing as well as the kSZ effect and density maps on the past light cone of an observer. Analyzing an ensemble of mocks, we find that the dipole field can be reconstructed with high fidelity over a range of angular scales and redshift bins. However, we present evidence for a bias due to the non-linear evolution of structure. We also analyze correlations with the primary CMB, investigating the ability of kSZ tomography to reconstruct the intrinsic CMB dipole. Our results constitute a proof-of-principle that $\mathrm{kSZ}$ tomography is a promising technique for future datasets.
\end{abstract}

\section{INTRODUCTION}

Fueled by rapid improvement on the experimental and data analysis fronts, our theoretical understanding of the Universe has condensed into the standard model of cosmology, $\Lambda$ CDM. This model is able to describe our Universe with a high degree of accuracy. Nevertheless, the fundamental nature of the major constituents of the model remains unknown, and a number of potential anomalies remain unexplained (see 1 for a recent review). The primary cosmic microwave background (CMB) has thus far been the workhorse of cosmology,

however the primary CMB temperature anisotropies have now been measured across an impressive range of angular scales to their ultimate cosmic variance limit by the Planck satellite. While additional progress can be made using measurements of CMB polarization, it will be necessary to cultivate additional observables to further improve.

One of the next frontiers of observational cosmology lies in the secondary CMB, temperature and polarization anisotropies induced by the scattering of CMB photons from mass (lensing) or free charges (the Sunyaev Zel'dovich effect). These effects are important on angular scales of roughly an arcminute, where the power in the primary CMB is rapidly falling due to Silk damping. In this paper, our primary focus is the kinetic Sunyaev Zel'dovich (kSZ) effect, temperature anisotropies generated by CMB photons scattering off of free electrons in bulk motion. The $\mathrm{kSZ}$ effect is the dominant blackbody contribution to the CMB on 
small angular scales. Although the amplitude of fluctuations is small, of order a microkelvin, the kSZ effect has now been detected at greater than $4 \sigma$ [2 [5], with future experiments [6] forecasted to achieve signal to noise in excess of $10^{2}$.

The kSZ effect can be expressed as the line of sight integral [7]

$$
\left.\frac{\Delta T}{T}\right|_{k S Z}\left(\hat{\mathbf{n}}_{e}\right)=-\sigma_{T} \int_{0}^{\chi_{r e}} d \chi_{e} a_{e}\left(\chi_{e}\right) \bar{n}_{e}\left(\chi_{e}\right)\left(1+\delta_{e}\left(\hat{\mathbf{n}}_{e}, \chi_{e}\right)\right) v_{\mathrm{eff}}\left(\hat{\mathbf{n}}_{e}, \chi_{e}\right),
$$

where $\sigma_{T}$ is the Thompson cross-section, $\bar{n}_{e}\left(\chi_{e}\right)$ is the average electron number density at comoving distance $\chi_{e}, \delta_{e}\left(\hat{\mathbf{n}}_{e}, \chi_{e}\right)$ is the electron overdensity field, $\hat{\mathbf{n}}_{e}$ is the angular direction on the sky to the electron, $v_{\text {eff }}\left(\hat{\mathbf{n}}_{e}, \chi_{e}\right)$ is the projection of the remote CMB dipole field (the CMB dipole observed by each electron along the line of sight), and $a_{e}$ is the scale factor at the scatterer's location.

The remote dipole field at any given point in spacetime depends on the local peculiar velocity of electrons as well as primordial contributions from the surface of last scattering (SLS). Because each electron samples a different portion of the SLS, the kSZ effect in principle contains more information about fluctuations on the largest scales than the primary CMB alone 8 18. However, much of this extra information is diluted by the line of sight integral in Eq. 1. presenting a challenge for making progress with the kSZ power spectrum alone.

Key to extracting information about the dipole field, and therefore the largest scales, is to use crosscorrelations with probes of large-scale structure (LSS) such as galaxy surveys and $21 \mathrm{~cm}$ measurements, a technique known as kSZ tomography 19 21. While a number of variants exist, we focus on direct crosscorrelations between the small angular scale $\mathrm{CMB}$ and the redshift-binned density field. A set of theoretical tools for kSZ tomography was developed in [17, and an optimal quadratic estimator derived in [18. Schematically, the correlation function is $\left\langle\Delta T_{\mathrm{kSZ}} \delta\right\rangle \sim\left\langle v_{\mathrm{eff}} \delta \delta_{e}\right\rangle \sim v_{\mathrm{eff}}\left\langle\delta \delta_{e}\right\rangle$ where $\delta$ is the overdensity field for the tracer. Importantly, since the dipole field receives contributions mainly from large scale modes, while the density fields receive contributions mainly from small-scale modes, the result is an isotropic power modulated by the dipole field. In analogy with reconstruction techniques for CMB lensing 22 and patchy reionization 23 , this statistical anisotropy is the basis for reconstructing the remote dipole field. By constructing correlators for each redshift bin, one can reconstruct the fully three-dimensional coarse-grained dipole field. Ref. [18] forecasted that a high fidelity reconstruction of the dipole field should be possible with next-generation galaxy surveys such as LSST [24] and next-generation CMB experiments such as CMB-S4 [6].

The goal of this paper is to further explore the reconstruction of the remote dipole field by analyzing a set of mock CMB and LSS maps generated from a suite of simulations. Such simulations allow us to explore previously neglected effects such as gravitational non-linearities, redshift space distortions, and CMB lensing. However, a proper treatment of all relevant physics is intrinsically challenging. One must model both the dipole field, which receives important contributions from scales of order the size of the observable Universe, as well as the density fields, which depend on small scales and include baryonic physics. Evolving a standard N-body simulation incorporating such a large range of scales is currently computationally intractable. To overcome this limitation, we develop a novel box-in-box simulation framework which consistently embeds a Gpc-sized N-body simulation inside of a box whose volume is larger than the observable Universe, and which contains large-scale modes evolved using linear theory. This box-in-box procedure allows us to use the data from both of these simulations to produce properly correlated maps of the lensed primary CMB temperature anisotropies, kSZ temperature anisotropies, the dipole field, and the dark matter overdensity field. We do not model baryonic physics in the present analysis, and therefore use the dark matter density as a proxy for the electron density. However, because our simulation framework is modular, N-body simulations including baryonic physics will be incorporated in the future.

We find that the quadratic estimator efficiently reconstructs the remote dipole field over a range of angular scales and redshift bins, indicating that kSZ tomography is generally robust. However, we present evidence for a bias due to non-linear structure at low redshifts. We demonstrate the ability of kSZ tomography to reconstruct the fundamental component of the observed CMB dipole, supporting the suggestion in previous work 18 that this could be an early application of kSZ tomography on large angular scales. The results we present here are intended primarily as a proof-of-principle both of the simulation framework and remote dipole reconstruction. To lay the groundwork for the analysis of near-term datasets, various layers of realism will be added to our simulation framework in future work, including the construction of mock galaxy catalogs, improved resolution, inclusion of baryonic physics, correlated foregrounds such as thermal SZ, and partial sky data. 


\section{THE REMOTE DIPOLE FIELD}

In this section we briefly present expressions for the remote dipole field, its theoretical power spectrum, and the real-space quadratic estimator that we use to reconstruct the dipole field. Further details on the velocity power spectra, transfer functions and harmonic-space quadratic estimators can be found in [17, 18. In order to work with a binned version of Eq. 1. we consider a bin-averaged remote dipole field $\bar{v}_{\text {eff }}^{\alpha}\left(\hat{\mathbf{n}}_{e}\right)$, which can be expressed in terms of contributions to the CMB temperature $\Theta\left(\hat{\mathbf{n}}_{e}, \chi_{e}, \hat{\mathbf{n}}\right)$ seen along the sky direction $\hat{\mathbf{n}}$ by free falling electrons at positions $\mathbf{r}_{e}=\chi_{e} \hat{\mathbf{n}}_{e}$ inside each redshift bin:

$$
\bar{v}_{\text {eff }}^{\alpha}\left(\hat{\mathbf{n}}_{e}\right)=\frac{3}{4 \pi} \frac{1}{\Delta \chi_{\alpha}} \int_{\chi_{\min }^{\alpha}}^{\chi_{\max }^{\alpha}} d \chi_{e} \int d^{2} \hat{\mathbf{n}} \Theta\left(\hat{\mathbf{n}}_{e}, \chi_{e}, \hat{\mathbf{n}}\right)\left(\hat{\mathbf{n}} \cdot \hat{\mathbf{n}}_{e}\right),
$$

Here, the index $\alpha$ labels each bin, which extend over the range in comoving distance $\chi_{\min }^{\alpha}<\chi<\chi_{\max }^{\alpha}$, and where $\Delta \chi_{\alpha}=\chi_{\max }^{\alpha}-\chi_{\min }^{\alpha}$. The radiation field is

$$
\Theta\left(\hat{\mathbf{n}}_{e}, \chi_{e}, \hat{\mathbf{n}}\right)=\Theta_{\mathrm{SW}}\left(\hat{\mathbf{n}}_{e}, \chi_{e}, \hat{\mathbf{n}}\right)+\Theta_{\mathrm{ISW}}\left(\hat{\mathbf{n}}_{e}, \chi_{e}, \hat{\mathbf{n}}\right)+\Theta_{\operatorname{Doppler}}\left(\hat{\mathbf{n}}_{e}, \chi_{e}, \hat{\mathbf{n}}\right),
$$

which receives contributions from the Sachs-Wolfe effect, the integrated Sachs-Wolfe effect due to the evolution of the gravitational potential along the line of sight, and the Doppler effect due to peculiar motion of electrons at $\mathbf{r}_{e}$ relative to the SLS (see e.g. 25]). The binned power spectrum is given by

$$
C_{\alpha \beta l}^{\bar{v} \bar{v}}=\int \frac{d^{3} k}{(2 \pi)^{3}} P_{\Psi}(k) \Delta_{\alpha l}^{\bar{v} *}(k) \Delta_{\beta l}^{\bar{v}}(k),
$$

where Greek indices denote redshift bins, $P_{\Psi}(k)$ is the power spectrum of the Newtonian gauge primordial gravitational potential $\Psi$, and $\Delta_{l}^{\bar{v}}\left(k, \chi_{e}\right)$ is the remote dipole transfer function, given in Ref. [18. As shown in [18, the presence of a large scale dipole will manifest in the cross correlation between the kSZ contribution to the CMB temperature and the moments of a redshift binned density distribution $\delta^{\alpha}$ defined by

$$
\delta^{\alpha}(\hat{\mathbf{n}})=\frac{1}{\Delta \chi_{\alpha}} \int_{\chi_{\min }^{\alpha}}^{\chi_{\max }^{\alpha}} d \chi \delta(\hat{\mathbf{n}}, \chi) .
$$

A real-space optimal quadratic estimator for the moments of the bin-averaged remote dipole field, $\widehat{v}_{\text {eff }, l m}^{\alpha}$, is given by:

$$
\begin{gathered}
\widehat{v}_{\mathrm{eff}, \ell m}^{\alpha}=N_{\alpha \ell}^{\bar{v} \bar{v}} \int d^{2} \hat{n} Y_{\ell m}^{*}(\hat{n}) \xi(\hat{n}) \zeta^{\alpha}(\hat{n}) . \\
\xi(\hat{n})=\sum_{\ell m} \frac{a_{\ell m}^{T}}{C_{\ell}^{T T}} Y_{\ell m}(\hat{n}) \\
\zeta^{\alpha}(\hat{n})=\sum_{\ell m} \frac{\delta_{\ell m}^{\alpha} C_{\alpha \ell}^{\delta \tau}}{C_{\alpha \ell}^{\delta \delta}} Y_{\ell m}(\hat{n})
\end{gathered}
$$

where

$$
\frac{1}{N_{\alpha l}^{\bar{v} \bar{v}}}=\frac{1}{2 l+1} \sum_{l_{1} l_{2}} \frac{\Gamma_{l_{1} l_{2} l \alpha}^{\mathrm{kSZ}} \Gamma_{l_{1} l_{2} l \alpha}^{\mathrm{kSZ}}}{C_{l 1}^{T T} C_{\alpha l_{2}}^{\delta \delta}} .
$$

The coupling constant $\Gamma_{l_{1} l_{2} l \alpha}^{\mathrm{kSZ}}$ is defined by

$$
\Gamma_{l_{1} l_{2} l \alpha}^{\mathrm{kSz}}=\sqrt{\frac{\left(2 l_{1}+1\right)\left(2 l_{2}+1\right)(2 l+1)}{4 \pi}}\left(\begin{array}{ccc}
l_{1} & l_{2} & l \\
0 & 0 & 0
\end{array}\right) C_{\alpha, l_{2}}^{\tau \delta},
$$

where the quantities with parenthesis are Wigner $3 \mathrm{j}$ symbols and $C_{\alpha, l_{2}}^{\tau \delta}$ is the cross-power between the binned galaxy density and the anisotropies in the optical depth of the redshift bin

$$
\tau^{\alpha}(\hat{\mathbf{n}})=-\sigma_{T} \int_{\chi_{\min }^{\alpha}}^{\chi_{\max }^{\alpha}} d \chi a(\chi) \bar{n}_{e}(\chi)\left(1+\delta_{e}(\hat{\mathbf{n}}, \chi)\right) .
$$

Because the simulations presented below do not contain baryons, we assume that the electron density field traces the dark matter density field. 


\section{SIMULATIONS}

Our simulation framework includes two components: a small-scale N-body simulation and a large-scale random field evolved using linear perturbation theory. We explore the idea of "sewing" these simulations together in order to accurately model physics on both large and small scales, thereby obtaining consistent realizations of both the primary $\mathrm{CMB}$ and angular, projected matter fields.

In order to obtain lightcone data on small scales, we use the publicly available L-PICOLA code [26. L-PICOLA is a "Lightcone-enabled Parallel Implementation of the COLA" method, providing an efficient means for generating both data on an observer's past lightcone and data on spatial hypersurfaces. The COmoving Lagrangian Acceleration ("COLA") method [27, 28] works by solving the second-order Lagrangian perturbation theory (2LPT) equations in order to generate an initial guess for the motion of particles in the simulation, and subsequently solves a set of equations describing the difference between the 2LPT solution and the full N-body equations in order to improve the accuracy of the 2LPT solution. This method allows L-PICOLA to obtain results with an accuracy similar to full N-body simulations on the scales we are interested in, but with a substantially larger simulation timestep, and therefore at a substantially reduced computational cost. In the limit of many timesteps, the output from L-PICOLA should be equivalent to a traditional N-body simulation.

Although these N-body simulations are able to provide us with particular realizations of physics on smallscales, we are interested in modeling both the primary CMB and kSZ temperature fields. In order to obtain contributions to the kSZ temperature from the full dipole field (Eq. 2) in a manner consistent with the small-scale L-PICOLA data, as well as to generate the primary CMB, we utilize a novel "box-in-box" technique. This technique is similar in spirit to the mode-adding procedure (MAP) described in 29, 30, in that information about large scales is added to a small-scale simulation. However, the technique we utilize differs in several important regards. Similar to [31, we add information at the level of the density and peculiar velocity fields directly in Eulerian or configuration space, rather than in either Fourier space or Lagrangian space; additionally, no information is removed from the small-scale simulation.

We utilize N-body simulations with a number of particles $N_{p}=1280^{3}$ in a comoving volume $(2 \mathrm{Gpc} / \mathrm{h})^{3}$, corresponding to a maximum simulation redshift of $z \sim 0.37$ and particle mass $2 \times 10^{12} \mathrm{M}_{\odot}$. While this coarse resolution does not allow us to resolve the structure of small mass halos, and also does not necessarily result in high-fidelity simulation data on the associated length scales, we find that the data we do obtain is sufficient for use in producing maps at angular resolutions of interest to us. We require the large-scale random field to encompass a volume containing the CMB (and ideally larger modes), so we utilize a large-scale "box" with volume $(32 \mathrm{Gpc} / \mathrm{h})^{3}$, resolved by $320^{3}$ grid points.

The "box-in-box" method should be valid in a regime similar to the MAP method, which itself has been shown to perform well when linear theory provides a good description of the field content. This is a somewhat stronger condition than requiring mode amplitudes or the power spectrum to be well-described by linear theory. While the linear and nonlinear matter power spectra agree to within a few percent down to scales of order $10 \mathrm{Mpc}$, mode coupling can exist - nonlinear terms of order $\delta \rho^{2}$ can constitute percent or larger corrections to evolution of the density field on scales of order a few hundred Mpc. So long as we remain in a regime where the field configuration is sufficiently well-described by (only) linear theory, we can expect the box-in-box technique to work. For the Gpc-scale N-body box sizes we employ here, this is the case.

\section{A. Simulating small scales using L-PICOLA}

We make use of both the lightcone output from L-PICOLA as well as data from spatial slices. The particle data from spatial slices is used to compute both primordial and large-scale components of the kSZ and primary CMB, and will be discussed in Section IIIB. The lightcone data is used to construct lightconeprojected sky maps of the density contrast field, velocity, and momentum fields, as well as convergence maps.

We generate radially binned maps of various fields, both in order to examine the underlying physics of the simulations, as well as to test reconstruction techniques at various redshifts. We divide the lightcone data into a number of radial bins between us and the largest redshift probed by the simulation. These radial bins can then be selectively integrated over to construct the contributions to a given field, such as density or kSZ temperature, from a given redshift range. 
In order to produce density maps in both radial and angular bins, we bin particle data by noting that

$$
\delta_{\text {bin }}=\frac{\rho_{\text {bin }}-\bar{\rho}_{\text {bin }}}{\bar{\rho}_{\text {bin }}}=\frac{n_{\text {bin }}}{\bar{n}_{\text {bin }}}-1
$$

where $\rho$ is the physical density inside a radial-angular-bin on the lightcone with comoving volume $V_{\text {bin }}=$ $\frac{\Omega_{\mathrm{bin}}}{3}\left(\chi_{B}^{3}-\chi_{A}^{3}\right)$, where the bin has radial boundaries at $\chi_{A}$ and $\chi_{B}$, and subtends a solid angle $\Omega_{\mathrm{bin}}$. The number of simulated particles of mass $m$ in a bin is $n=\rho / m$, and the average/expected/background number of particles in a pixel is

$$
\bar{n}_{\text {bin }}=N_{\text {sim }} \frac{V_{\text {bin }}}{V_{\text {sim }}}=\frac{N_{\text {sim }}}{V_{\text {sim }}} \frac{\Omega_{\text {bin }}}{3}\left(\chi_{B}^{3}-\chi_{A}^{3}\right)
$$

in the case of discrete bins, or

$$
\bar{n}_{\text {bin }}=\frac{N_{\text {sim }}}{V_{\text {sim }}} \frac{\Omega_{\text {bin }}}{3} 3 \chi^{2} d \chi
$$

in the continuum limit, with $N_{\text {sim }}$ the total number of particles in a simulation of comoving volume $V_{\text {sim }}$. The overdensity is then given by taking $n_{\text {bin }}$ to be the number of particles in a given bin, so explicitly,

$$
\delta_{\text {bin }}=-1+\sum_{\text {particles } \in \text { bin }} \frac{1}{\bar{n}_{\text {bin }}} .
$$

This expression is similar in spirit to that of [32, although not identical. We also integrate the density contrast along a line of sight - or in a pixel subtending some solid angle on the sky; this can be written as a sum over the densities of all bins along the line of sight of the pixel,

$$
\delta=\int d \chi \delta(\chi)=\sum_{\text {bin } \in \text { pix }} \delta_{\text {bin }} d \chi_{\text {bin }}
$$

where the bins that lie along the direction of the pixel on the sky are summed over. For different choices of radial binning, the sum will agree up to terms $\mathcal{O}\left(d \chi^{2}\right)$.

We are additionally interested in accounting for redshift-space distortions (RSDs) within this framework, requiring a small modification to the density field used in the reconstruction, Eq. 5 . In order to take RSDs into account, we perturb particle positions by a small amount corresponding to the mis-inferred distance. Quantitatively, we compute

$$
\chi_{\mathrm{RSD}}=\chi_{\mathrm{FRW}}\left(z_{\mathrm{FRW}}(\chi)+v_{\text {Doppler }}\right)
$$

where functions with the FRW subscripts indicate the background FRW cosmology has been used, and where we then bin particles using Eq. 13 but according to their position $\chi_{\mathrm{RSD}}$. During the later discussion of reconstruction in this paper, the density field used in reconstruction is the one that accounts for RSDs.

The convergence, formally written as

$$
\kappa=\frac{3}{2} H_{0}^{2} \Omega_{m, 0} \int_{0}^{\chi_{\mathrm{CMB}}} d \chi \frac{\chi\left(\chi_{\mathrm{CMB}}-\chi\right)}{\chi_{\mathrm{CMB}}} \frac{\delta(\chi)}{a(\chi)},
$$

can similarly be binned. An expression for convergence binned in discrete angular pixels that is independent of radial binning is used [33, 34, allowing contributions to be placed into radial bins that can be summed over later to examine the convergence contribution from a given radial bin or range of radial bins,

$$
\kappa_{\text {bin }}=\frac{3}{2} H_{0}^{2} \Omega_{m, 0} \frac{V_{\mathrm{sim}} / N_{\mathrm{sim}}}{\Omega_{\text {bin }}} \sum_{\text {particles, } p, \in \text { bin }} \frac{1}{\chi_{p} a\left(\chi_{p}\right)} \frac{\chi_{\mathrm{CMB}}-\chi_{p}}{\chi_{\mathrm{CMB}}},
$$

so that for each angular pixel on the sky the total convergence will be

$$
\kappa_{\text {pix }}=\sum_{\text {bin } \in \text { pix }} \kappa_{\text {bin }} .
$$


We use this convergence map to lens the primary CMB.

There are several ways to compute the kSZ temperature fluctuations from particle data. The kSZ temperature fluctuations given by Eq. 1 can be evaluated by binning the components of the fields $v$ and $\delta$ separately. However, the peculiar velocity field can be severely undersampled in simulated data, with nonzero velocities determined by only a single particle, or not at all in some pixels. In the case of the density field the issue is not as severe, as a lack of particles is merely indicative of an underdense region, where the density should be small anyways. A standard practice is therefore to write the integral in terms of a sum over peculiar particle momenta [35],

$$
\left(\frac{\Delta T}{T}\right)_{k S Z}=-\frac{\sigma_{T} f_{b} \mu}{\Omega_{\text {pix }}} \sum_{\text {particles } p \in \text { bin }} \frac{m_{p} v}{D_{A, p}^{2}}
$$

In standard techniques used to construct kSZ temperature maps, the only contribution to the temperature field considered is the peculiar velocity of matter in Newtonian gauge projected along the line of sight, $v=v_{\text {Doppler, } \mathrm{N}-\text { body }}$. Thus, important contributions to the observed $\mathrm{kSZ}$ temperature perturbations on large angular scales from ISW, SW, or large-scale velocity modes (modes larger than the simulation volume) have not been modeled, each of which will contribute to the kSZ temperature fluctuations as described by Eq. 3 .

In Section IIIB we discuss more precisely how we model these additional contributions, however at the level of binning, we have two options. We can include these fields at the level of the already-binned lightcone data, replacing $v$ with

$$
v \rightarrow v_{\text {eff }}=v_{\text {Dopp }, \mathrm{N}-\text { Body }}+v_{\text {Dopp }, \mathrm{LS}}+v_{\text {ISW }}+v_{\mathrm{SW}}
$$

where the ISW and SW components are given by the respective contributions of the effects (Eq. 3) to the temperature perturbation (Eq. 1), and where the Doppler contributions are from both the N-body simulation and large-scale (LS) modes not included in the N-body simulation. Alternatively, we can compute the overdensity $\delta_{\text {bin }}$ and the velocity $v_{\text {eff }}^{\text {bin }}$ in each bin, and evaluate Eq. 1 directly. We find that both methods result in nearly identical $\mathrm{kSZ}$ temperature maps and power spectra for the angular resolutions we are interested in, although for the final maps we use Eq. 19 and 20.

The final quantity we compute using lightcone data is the peculiar velocity field, taking the velocity in each bin to simply be the average velocity of particles within each bin. At low angular resolutions, which for our simulations means a HEALPIX [36] resolution of Nside $=1024$, the narrowest redshift-angular bins we consider will typically contain at least one particle. At higher resolutions, artifacts become apparent in velocity maps due to undefined velocities in cells without particles [37. However, for the dipole field in particular, it is sufficient to compare low-Nside maps to our reconstructed velocity maps as we are interested in reconstructing the dipole field on large angular scales $(\ell \lesssim 20)$.

As a final point of note, and as a check that the temperature maps and especially the dipole field reconstruction is insensitive to the precise binning method used, we employ binning using both a "nearest gridpoint" assignment scheme, and a "cloud-in-cell"-type assignment scheme where contributions from individual particles of fields are distributed to a weighted average of nearby cells, both radially and in an angular direction. The latter of these methods introduces additional smoothing, or aliasing, on bin-sized scales; this suppresses power on these scales, but also suppresses the effects of shot noise. Despite this difference, we find that the performance of the dipole field reconstruction presented below is largely insensitive to this detail.

\section{B. Large scales: "box-in-box"}

We formally describe the process of sewing the N-body data and the large-scale modes together using a "coloring" operator $\mathcal{C}_{P_{c}}(f)$ that rescales a stochastic field $f$ (with its own power spectrum $P_{f}$ ) by a power spectrum $P_{c}$,

$$
\mathcal{C}_{P_{c}}(f)=\int \frac{d^{3} k}{(2 \pi)^{3}} e^{i \vec{k} \vec{x}} f(\vec{k}) P_{c}^{1 / 2}(k)
$$

so the power spectrum of the resulting field is given by $P_{f} P_{c}$. For a coloring spectrum $P_{c}=P_{f}^{-1}$, the field will be whitened. We additionally make use of an "inlay" operator, $\mathcal{I}\left(f_{1}, f_{2}\right)$, which acts in configuration 
space to replace values in the interior of a (large-scale) field by values of a second (coarsened, small-scale) field. The procedure of sewing a small field into another larger field then consists of the following operations:

$$
f_{\text {sewn }}=\mathcal{C}_{P_{f}}\left(\mathcal{I}\left(\mathcal{C}_{P_{f}^{-1}}\left(f_{\mathrm{lg}}\right), \mathcal{C}_{P_{f}^{-1}}\left(f_{\mathrm{sm}}\right)\right)\right)
$$

Evaluating the above expression entails taking Gaussian random fields $f_{\mathrm{sm}}$ and $f_{\mathrm{lg}}$, both with statistical properties described by $P_{f}$, whitening these fields, replacing values of the lg field by ones from the sm field, and finally de-whitening the fields.

The result of this procedure on the lg field is that the small-scale modes in the region of replacement are now given by modes from the sm field, while large-scale modes have been preserved and superimposed upon the small-scale field.

Although L-PICOLA provides us with information about the density field, we are ultimately interested in obtaining the primordial potential, from which we can compute corrections to the velocity field using linear theory. In order to obtain the primordial potential on large scales, we extract the potential on the initial slice using the density field and Poisson equation ${ }^{1}$,

$$
\nabla^{2} \Phi=4 \pi G a^{2} \delta_{\rho}^{\operatorname{sim}} .
$$

The potential can then be evolved back in time using the transfer function for the potential, $T\left(\phi_{\mathrm{sim}} \rightarrow \phi_{\text {prim }}\right)$.

We are therefore interested in computing

$$
\phi_{\text {sewn }}=T\left(\phi_{\text {sim }} \rightarrow \phi_{\text {prim }}\right) \frac{4 \pi G a^{2}}{\nabla^{2}} \mathcal{C}_{P_{\delta}}\left(\mathcal{I}\left(\mathcal{C}_{P_{\delta}^{-1}}\left(\delta_{\text {Box }}\right), \mathcal{C}_{P_{\delta}^{-1}}\left(\delta_{\mathrm{LP}}\right)\right)\right)
$$

where the L-PICOLA density field is noted by the LP subscript, and the Box subscript refers to a random realization of a density field with power spectrum $P_{\delta}$. Written in Fourier space, the outermost coloring operation, transfer function operation, and the inverse Laplacian operation can all be combined into an operation equivalent to coloring by the primordial spectrum. Coloring the large-scale Box modes with its inverse spectrum is also equivalent to simply generating a field of white noise, $N_{\text {white }}$. Thus the final operation we perform in order to obtain a large-scale primordial potential consistent with the density field from the L-Picola simulation is

$$
\phi_{\text {sewn }}=\mathcal{C}_{P_{\phi, \text { prim }}}\left(\mathcal{I}\left(N_{\text {white }}, \mathcal{C}_{P_{\delta}^{-1}}\left(\delta_{\mathrm{LP}}\right)\right)\right.
$$

The power spectra for the density and primordial potential are both given by the CLASS code [41. We show snapshots of various steps of this procedure in Fig. 1]

Once we have the primordial potential, we use the CMB radiation transfer functions to obtain the primary $\mathrm{CMB}$, and velocity transfer functions to compute the contributions to the dipole field due to large-scale modes $^{2}$. From the large-scale modes, we can then compute the contributions to $v_{\text {eff }}$ from Eq. 20, and thus their contribution to the observed $\mathrm{kSZ}$ temperature fluctuations. When computing large-scale contributions to $v_{\text {eff }}$, we also need to ensure we do not double-count modes already accounted for by the N-body simulation. Therefore, when computing the large-scale Doppler contribution to $v_{\text {eff }}$, we only integrate over modes with wavelengths larger than the N-body simulation volume.

The CMB multipoles are then computed using the large-volume simulated primordial potential up to $\ell=28$. In principle we could generate additional CMB modes using simulated data, however they will not be correlated with the remote dipole field or the density field. We therefore use a random realization of the primary CMB $a_{l m} \mathrm{~s}$ at $\ell>28$, based on the theoretical power spectrum obtained from CLASS.

\footnotetext{
${ }^{1}$ As a technical note, we can safely interpret output from the L-PICOLA simulations in a standard way without worrying about relativistic effects given our accuracy requirements for the scales we are interested in 38, 40. The evolution of large-scale modes is determined using linear cosmological perturbation theory, which takes into account relativistic effects at linear order in metric and density perturbations.

${ }^{2}$ We could also modulate large-scale modes in the density field, however long-wavelength density perturbations contribute negligibly to the cross correlation between the kSZ temperature and density field [17, so we do not include this modulation.
} 

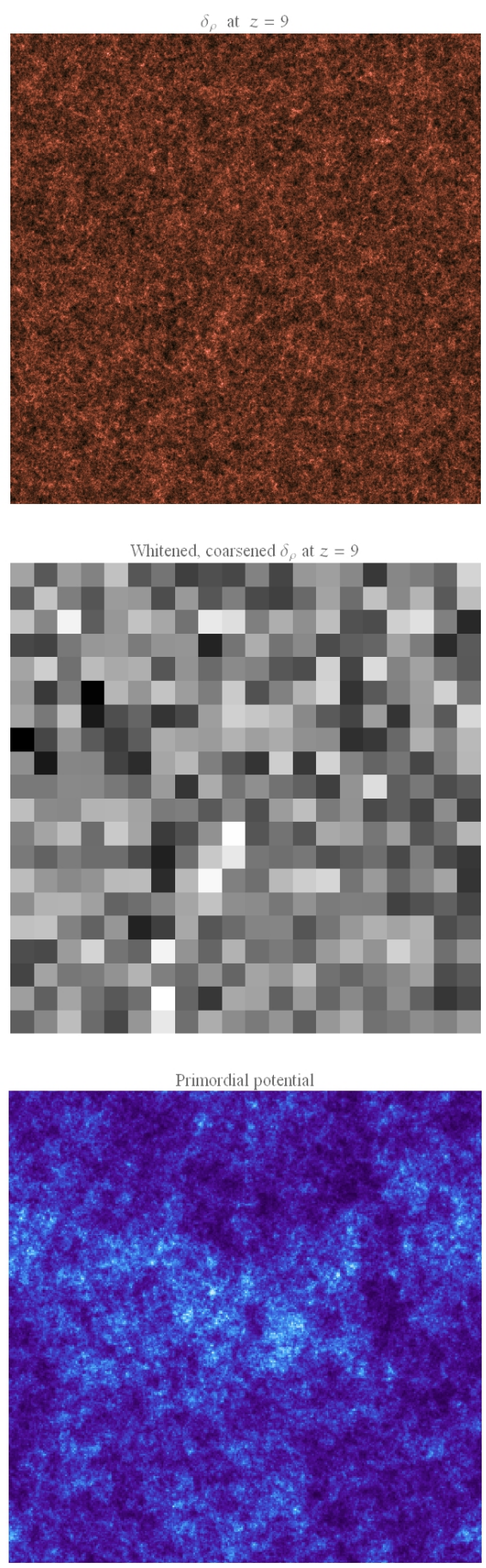

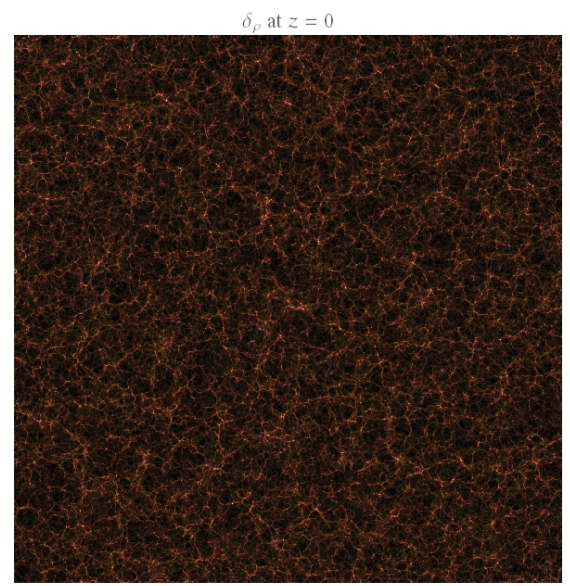

Random white noise field

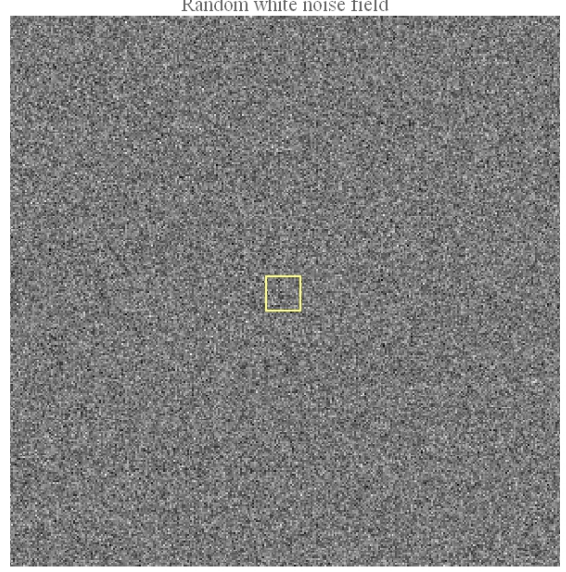

Potential with inlay subtracted (zoomed in

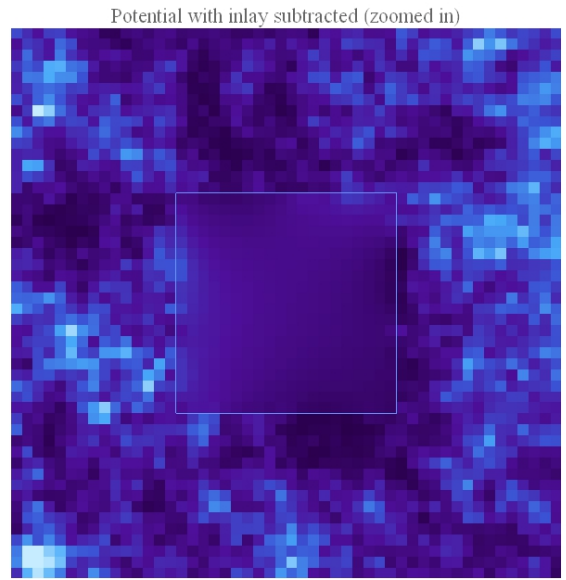

FIG. 1: Slices of spatial hypersurfaces of simulations during various parts of the sewing-in procedure described by Eq. 25 Top left: the initial L-Picola density contrast field at $z=9$, with comoving box size $L=2 \mathrm{Gpc} / h$. Top right: the density field at $z=0$. Middle left: the initial $z=9$ density field, whitened using the matter power spectrum, averaged over (coarsened) so the resolution is the same as that of the box containing large-scale modes. Middle right: A random realization of white noise for large-scale modes, with $L=32 \mathrm{Gpc} / h$. The central $2 \mathrm{Gpc} / h$ region that will be replaced has been outlined with a yellow border. Bottom left: The primordial potential with white-noise values in the large box replaced using the whitened L-PICOLA field, then colored using the primordial power spectrum. Bottom right: The central $5 \mathrm{Gpc} / h$, with the colored small-scale box values directly subtracted. Small residual large-scale modes can be seen in the center. The region where the subtraction has been performed is outlined. 
We also include lensing of the primary $\mathrm{CMB}$, utilizing the convergence maps generated from the lightcone data. From the maps we can compute the lensing potential $\phi$ in harmonic space as

$$
\phi_{\ell m}=\frac{2 \kappa_{\ell m}}{\ell(\ell+1)}
$$

The lensed CMB temperature is then given by

$$
T(\hat{n}) \rightarrow T(\hat{n}+\nabla \phi) \simeq T(\hat{n})+\nabla \phi \nabla T(\hat{n}) .
$$

Although the CMB is lensed, the $\mathrm{kSZ}$ temperature is not. In principle, there could be lensing of the $\mathrm{kSZ}$ temperature fluctuations due to any structures between $\mathrm{kSZ}$ sources and an observer, however we do not model this. The lensing we compute is also derived from only the N-body volume we simulate, thus in a more realistic treatment, structure at higher redshifts and on large scales would need to be included. However, the small-scale density-temperature correlations induced by lensing from the density field we use for reconstruction are accounted for. In future work, we would nevertheless prefer to include lensing (and $\mathrm{kSZ}$ ) contributions from additional redshifts.

To model kSZ temperature anisotropies sourced at redshifts beyond our N-body simulation, we include Gaussian random noise on angular scales $\ell \gtrsim 1000$ with amplitude $\sim 2 \mu \mathrm{K}$.

In Figure 2, we show various outputs of our simulation pipeline. Of particular note is that the that the projected density is properly correlated with the convergence field and the kSZ temperature anisotropies, and the dipole field is properly correlated with the primary $\mathrm{CMB}$ and the $\mathrm{kSZ}$ temperature anisotropies. Focusing on the kSZ map, both the large-scale contributions and large-scale modulation of power from the dipole field are visible. In addition, the primordial components of the dipole field (i.e. contributions from modes in the big box) are visible as responsible for the structure of the dipole field on large angular scales.

\section{RESULTS}

\section{A. Reconstruction using a quadratic estimator}

We now analyze data from an ensemble of ten simulations to assess the performance of the quadratic estimator, Eq. 6. We utilize two radial binning schemes, with the density field on the light cone of each simulation arranged into either a single bin or eight bins of equal radial comoving width. For each simulation and bin we construct maps of $\xi$ defined in Eq. 7 and $\zeta^{\alpha}$ defined in Eq. 8 . The power spectra $C_{\ell}^{T T}, C_{\alpha \ell}^{\delta \delta}$, and $C_{\alpha \ell}^{\delta \tau}$ used in Eqs. 7, 8,9 to generate the $\xi, \zeta^{\alpha}$ fields and reconstruction noise are the sample variances from each realization. We then obtain the estimated moments of the binned dipole field from Eq. 6, and generate a map of the reconstructed average dipole field in each bin.

In Figure 3 we compare the reconstructed and actual bin-averaged dipole fields for a single bin and for the 8 th bin of the eight bin configuration. All maps are filtered to contain only multipoles $\ell<28$. "By-eye," the reconstruction performs well on large angular scales. We quantify the agreement between the reconstructed and actual dipole field in two ways.

First, we make a comparison at the level of the power spectra in Fig. 4. We compute the mean and standard deviation of the reconstructed dipole field power (with the noise bias removed) and the actual dipole field power (total, and separate contributions from the small and big box modes), as well as the prediction from linear theory using Eq. 4. In this figure, we plot these quantities for the single bin (top left) and bins 2, 4 , and 8 of the eight bin configuration. In general, the agreement between the mean reconstructed and the mean actual power is quite good at low multipoles, within a single standard deviation. For higher multipoles, the reconstruction is poor and there is an excess of power due to the reconstruction noise. In addition, there appears to be a systematic bias towards extra power in the reconstructed field at low multipoles, especially in the single-bin configuration and the lowest redshift bins of the eight bin configuration; the agreement with linear theory becomes better at higher redshift. This is consistent with a bias due to gravitational nonlinearities, which we expect to be more important at low redshift. A similar bias exists in CMB lensing [42], and we hope to investigate this possibility in future work.

As an additional diagnostic of the performance of the reconstruction, we compute the reconstruction 

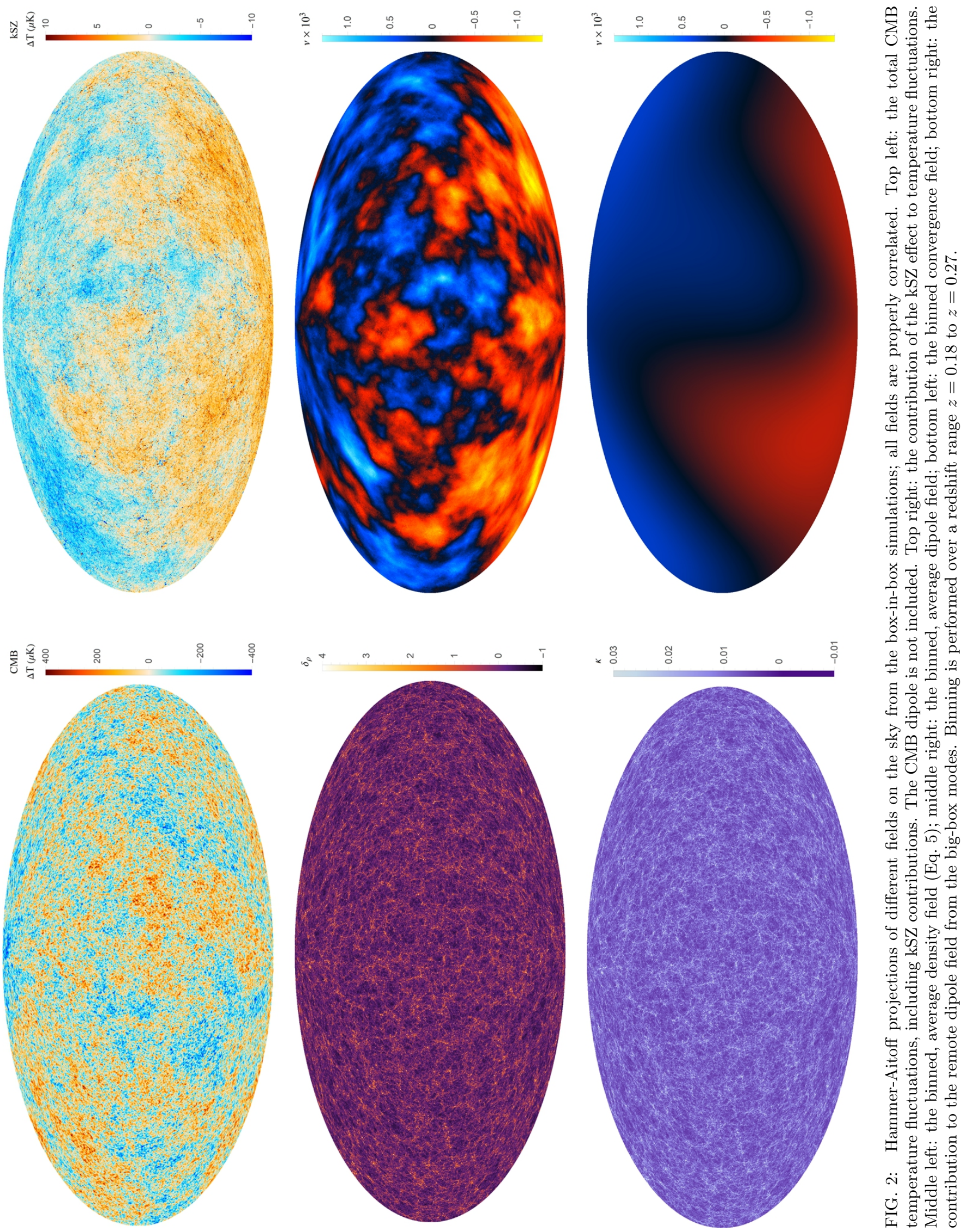

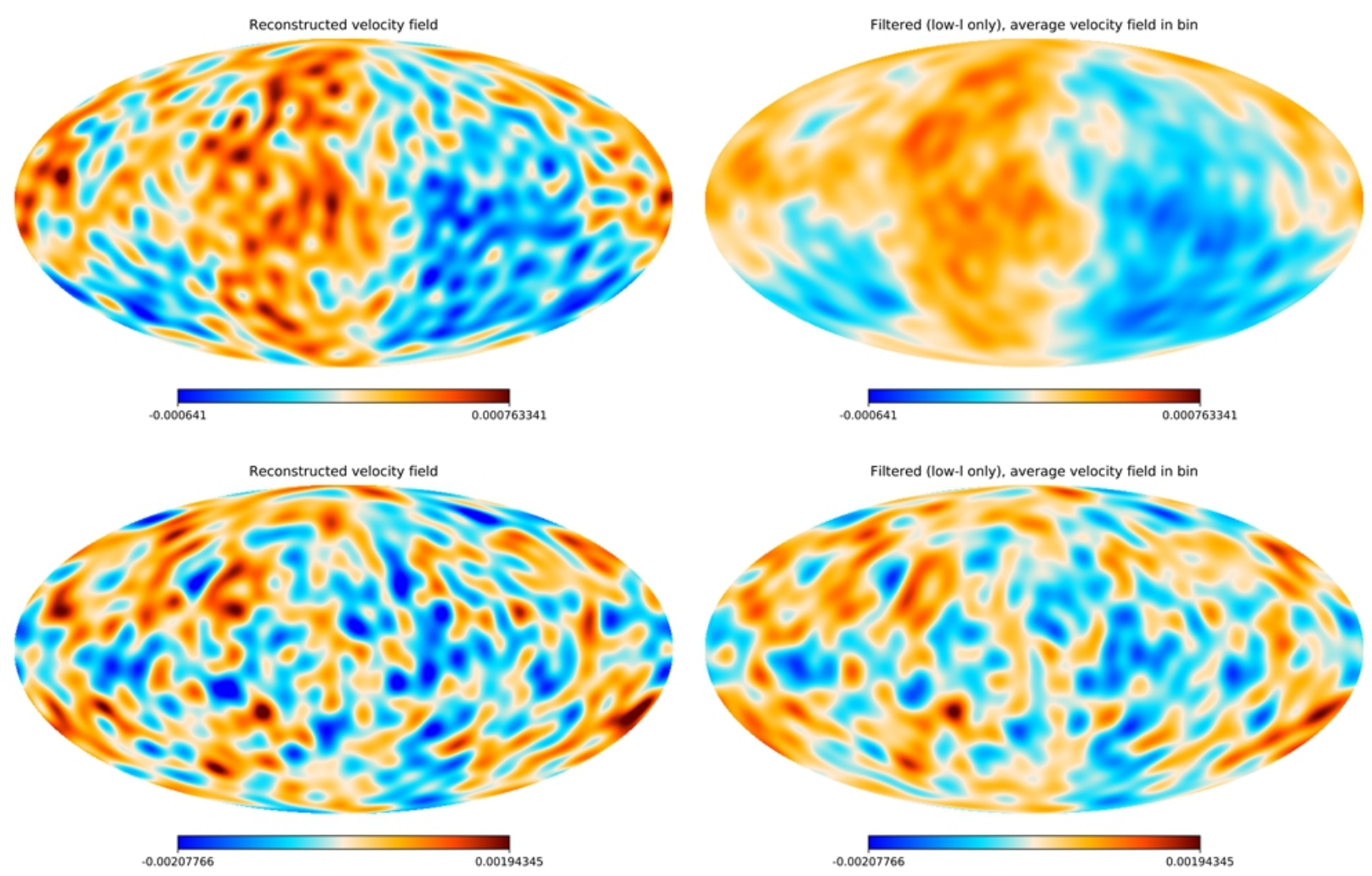

FIG. 3: The remote dipole field obtained from simulations compared to the reconstructed remote dipole field. The maps do not include modes higher than $\ell>28$. The reconstruction of the top two plots was done using a single redshift bin from $z=0.086$ to $z=0.37$, while the bottom plots are a redshift bin from $z=0.33$ to $z=0.37$. By eye, it is noticeable that large angular modes between the two maps tend to agree, while smaller-scale modes only do to a moderate extent. The reconstruction of smaller scales is also found to be better in the smaller, higher-redshift bin. This is in agreement with results obtained by looking at the reconstruction efficiency, shown in the top left panel of Figure 5 Excess power can also be seen on small scales, consistent with the spectra found in Figure 4.

efficiency

$$
r_{L} \equiv \frac{\hat{C}_{L}^{\hat{v} \bar{v}}}{\left(\hat{C}_{L}^{\hat{v} \hat{v}} \hat{C}_{L}^{\bar{v} \bar{v}}\right)^{1 / 2}},
$$

where $\hat{v}$ denotes the reconstructed field and $\bar{v}$ the actual field. The efficiency is not sensitive to an overall change in normalization, but instead provides us with a measure of how strongly correlated reconstructed and simulated modes are. In general, we find that the reconstructed modes agree well with the simulated modes on the largest angular scales. The reconstruction efficiency is found to be better at higher redshift, again we expect this due to a lack of nonlinear effects. Reconstruction is also found to perform better in smaller bins, an effect we can at least partially attribute to the increased information content: information from small-scale modes has not been so heavily averaged away. However, in larger redshift bins, the correlation with primordial modes is larger, as discussed in the next section.

\section{B. CMB-kSZ Dipole Correlation}

We now consider how well we can determine the intrinsic CMB dipole using information from the reconstructed large-scale velocity field, as suggested in Ref. [18. This idea is not without ambiguity - because one can arbitrarily change the CMB dipole by performing a boost, there is no unique definition of the intrinsic 

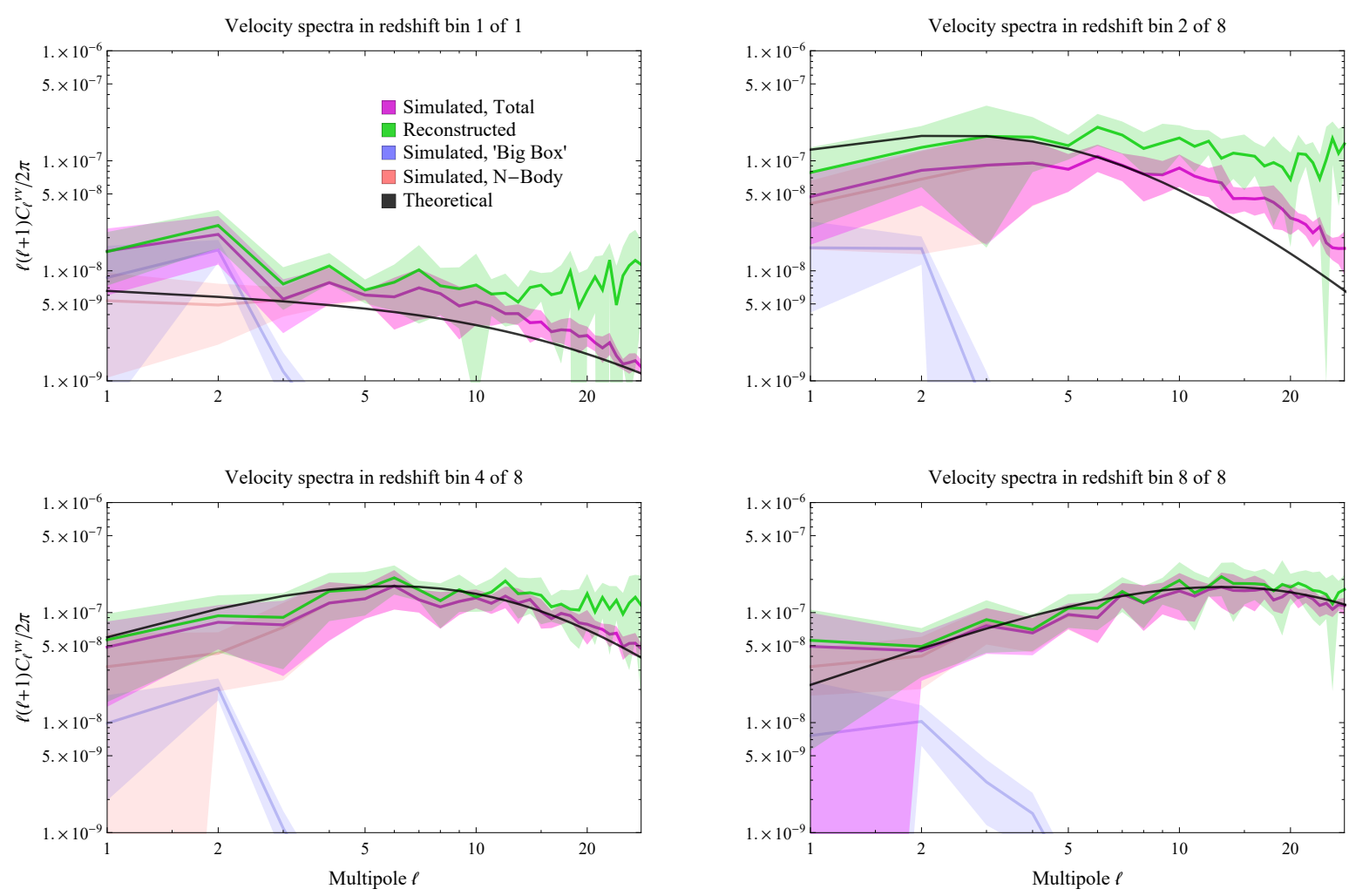

FIG. 4: The velocity power spectra from simulated data, compared to the theoretical and reconstructed spectra. Contributions to the spectra from the N-body simulation are shown in red, contributions from the large-scale box modes in blue, and the total in purple. The reconstructed spectra with noise subtracted is in green, and linear theory prediction in black. Lines indicate the mean spectrum from our simulations, while solid bands indicate the variance. The reconstruction is performed using redshift data in bins over a redshift range of $z=0.086$ to $z=0.37$, subdivided into one or eight bins of equal comoving distance. Reconstruction efficiencies are shown in Figure 5

dipole. Instead, one must settle on a definition universal and specific enough to facilitate a meaningful comparison. We can make progress by noting that the local CMB dipole should, to an extent depending on one's definition of the intrinsic CMB dipole, be correlated with the $\ell=1$ moments of the remote dipole field. The contributions to our measured CMB dipole and the remote dipole field of a nearby observer are determined primarily by small-scale modes which source local peculiar velocities. However, there are also subdominant contributions to the CMB dipole from larger-scale (but still local) velocity modes and other effects both along our past lightcone and at the CMB last scattering surface.

A standard definition of the fundamental $\mathrm{CMB}$ dipole is obtained by boosting to a reference frame in which the relativistic aberration of the CMB vanishes (see e.g. [43]). In Newtonian gauge, this aberrationfree dipole is calculated in the frame where an observer has vanishing local peculiar velocity, altering the Doppler term in Eq. 3 A more general definition of the fundamental CMB dipole is obtained by applying a low-pass filter to the Fourier modes contributing to local peculiar velocities. The aberration-free dipole is a special case, where all modes contributing to the local Doppler term are filtered out. This more general definition is also more closely related to the dipole field obtained in kSZ tomography, since the bin-averaging effectively imposes a low-pass filter on radial peculiar velocities. We will refer to this as the large-scale Doppler dipole.

We can quantitatively express the correlation between the remote dipole field and the various definitions of the $\mathrm{CMB}$ dipole in terms of transfer functions, with the CMB transfer function filtered below a given scale $k_{\text {cut }}$,

$$
C_{\alpha 1}^{T \bar{v}}=\int \frac{d^{3} k}{(2 \pi)^{3}} P_{\Psi}(k) \Delta_{\alpha 1}^{\bar{v} *}(k) \Delta_{\text {filt }, 1}^{T}(k),
$$

where as before, $\alpha$ labels a redshift bin in which the remote dipole field $\bar{v}$ is averaged. The filtered CMB 

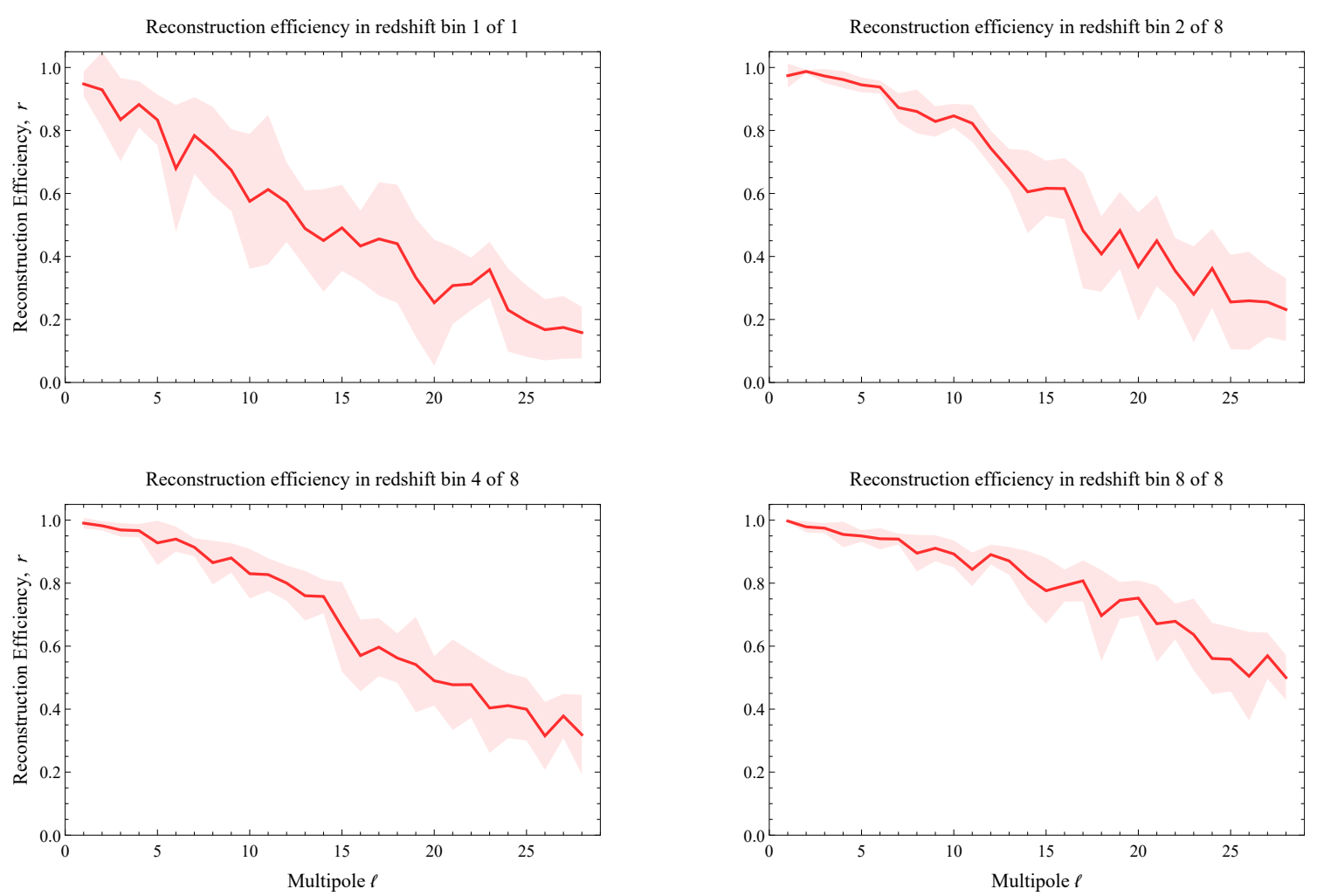

FIG. 5: The reconstruction efficiency for the runs in Figure 4 The solid line indicates the mean reconstruction efficiency in each bin for each simulation realization, and the solid band the standard deviation.

transfer function for the dipole is given by

$$
\Delta_{\text {filt }, 1}^{T}=\Theta\left(k_{\text {cut }}-k\right) \Delta_{\text {dopp, local }, 1}^{T}(k)+\Delta_{\text {dopp }, \mathrm{CMB}, 1}^{T}(k)+\Delta_{\mathrm{ISW}, 1}^{T}(k)+\Delta_{\mathrm{SW}, 1}^{T}(k)
$$

where $\Theta$ is the Heaviside step function, and the individual contributions to the radiation transfer function include ISW, SW, and both local and last-scattering-surface (CMB) Doppler contributions. For the largescale Doppler dipole, we choose a filtering scale equal to the N-body simulation volume $\left(L_{\text {cut }} \sim 2 \pi / k_{\text {cut }} \sim\right.$ $3 \mathrm{Gpc}$ ). For the aberration-free dipole, $k_{\text {cut }} \rightarrow \infty$.

In Figure 6, we plot the theoretical prediction for the correlation coefficient (e.g. Eq. 28) using linear theory between the $\ell=1$ moment of the bin-averaged remote dipole field and three definitions of the CMB dipole: the observed CMB dipole ("all Doppler"), the aberration-free dipole, and the large-scale Doppler dipole. We plot the theory prediction for a single bin of varying radial extent in redshift. In addition, we show the mean and standard deviation of the correlation coefficient calculated from ten simulations for redshift bins of two different size using the simulated CMB large-scale-filtered dipole and the reconstructed dipole field. As expected from the discussion above, the correlation between the observed CMB dipole and the bin-averaged dipole field is small for all but the smallest bins. Because they are composed primarily of large-scale modes, the correlation between the bin-averaged dipole field and the aberration-free and largescale Doppler dipoles improves with bin width. However, the dipole field has a finite correlation length, and therefore the correlation coefficient eventually goes down. We find that the large-scale Doppler dipole can in principle be determined with a maximum correlation coefficient of $r \sim 0.9$ while the aberration-free dipole can be determined with a maximum correlation coefficient of $r \sim 0.65$. The optimal reconstruction bin width corresponds to a redshift of $z \sim 0.4$. In conclusion, our simulations indicate that constraints on the intrinsic CMB dipole should reasonably be attainable in individual realizations. 


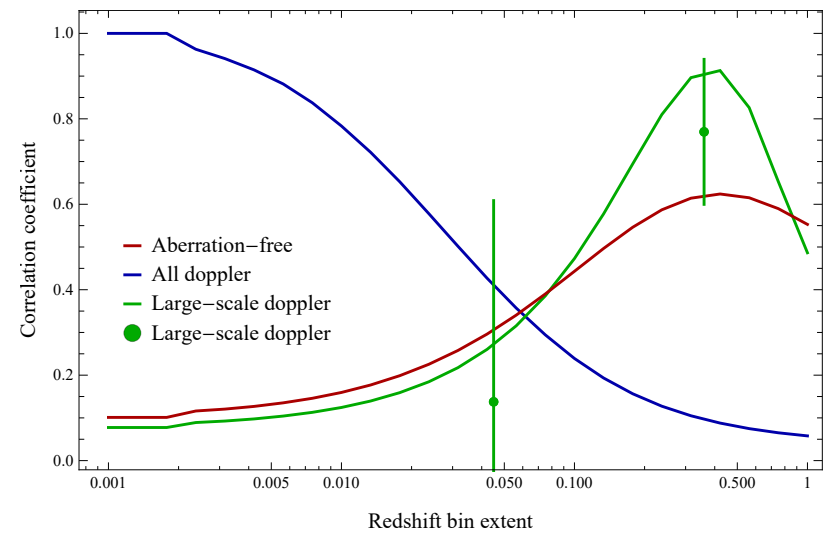

FIG. 6: The reconstructed velocity field and CMB temperature dipole correlation coefficient, $C_{1}^{T \bar{v}} / \sqrt{C_{1}^{T T} C_{1}^{\bar{v} \bar{v}}}$, computed using different CMB dipoles. The theoretical correlation using the full CMB transfer function is shown in blue, correlation with the aberration-free dipole in red, and the correlation with "filtered" CMB dipole shown in green. Data point show the correlation of the simulated CMB dipole filtered on 3 Gpc (box-sized) scales for two redshift bin sizes. The points are the mean correlation from all simulations we perform, and error bars denote the standard deviation.

\section{DISCUSSION AND CONCLUSIONS}

kSZ tomography is a useful tool for probing the largest observable scales in our Universe, providing information in addition to what the primary CMB and large-scale density surveys alone can tell us. In this work we have explored the ability of a quadratic estimator to reconstruct the remote dipole field using simulated maps of the $\mathrm{CMB}$ and density field. We have found that the reconstruction process is able to capture highly significant information about large scales, even in the presence of physical effects with the potential to contaminate our ability to reconstruct, including nonlinear growth of structure, RSDs, lensing, and contributions to the $\mathrm{kSZ}$ temperature from structures outside the range of redshifts considered for reconstruction.

We have accomplished this using a novel simulation technique, in which a small-scale N-body simulation is sewn into a large-scale volume evolved with linear theory, allowing us to generate self-consistent maps of $\mathrm{kSZ}$ temperature fluctuations, the primary $\mathrm{CMB}$, CMB lensing, density, and dipole fields. In turn, the consistency of these components allows us to explore the ability of reconstruction techniques to probe fundamental physics such as determining the intrinsic CMB dipole. Forthcoming work will additionally allow us to asses the ability of kSZ measurements to constrain parameters of cosmological models, especially important in the context of theories competing to describe dark energy and dark matter, and the presence of unexplained anomalies in the measured CMB.

While this work furthers our confidence in the ability of the reconstruction procedure to work in practice, it will be important to incorporate additional physics into our models in order to make future predictions as realistic and robust as possible. The presence of foregrounds may hamper our ability to reconstruct the remote dipole field; it will be necessary to ensure we can adequately clean thermal Sunyaev Zel'dovich emissions, a foreground strongly correlated with the density field.

We have also relied on several physical assumptions that can potentially affect the results presented here. One assumption is that the electron field directly traces the dark matter density field, something that in practice has been shown to fail on length scales below $\sim 10 \mathrm{Mpc}$ [4]. Research precisely modeling the impact of physics at play on small scales is ongoing, however we have found that the angular resolution requirements for accurate reconstruction of low- $\ell$ modes of the remote dipole field are fairly mild, thus the physical scales resolved by the simulations are as well.

Observations will also not provide perfect information about the density field as assumed in this study; rather, this information is typically obtained through direct observations, such as of galaxies and clusters at low redshift, or the $21 \mathrm{~cm}$ signal at higher redshifts. Accounting for these effects in angular maps of the density field can be accomplished in a future study in which we generate mock galaxy catalogs. In principle, existing data of this type can be combined with the box-in-box technique we introduce in order to produce 
maps of increasing realism.

\section{ACKNOWLEDGMENTS}

We would like to thank Neal Dalal, Moritz Munchmeyer and Marcel Schmittfull for helpful discussions. We would also like to thank Tom Giblin for discussions and for his help in accessing computational resources. The simulations in this work made use of hardware provided by the National Science Foundation and the Kenyon College Department of Physics. This research was supported in part by Perimeter Institute for Theoretical Physics. Research at Perimeter Institute is supported by the Government of Canada through the Department of Innovation, Science and Economic Development Canada and by the Province of Ontario through the Ministry of Research, Innovation and Science. MCJ was supported by the National Science and Engineering Research Council through a Discovery grant. JBM acknowledges support as a CITA National Fellow.

[1] D. J. Schwarz, C. J. Copi, D. Huterer, and G. D. Starkman, "CMB Anomalies after Planck," Class. Quant. Grav. 33 (2016) no. 18, 184001, arXiv:1510.07929 [astro-ph.C0]

[2] N. Hand et al., "Evidence of Galaxy Cluster Motions with the Kinematic Sunyaev-Zel'dovich Effect," Phys. Rev. Lett. 109 (2012) 041101, arXiv:1203.4219 [astro-ph.C0].

[3] F. De Bernardis et al., "Detection of the pairwise kinematic Sunyaev-Zel'dovich effect with BOSS DR11 and the Atacama Cosmology Telescope," JCAP 1703 (2017) no. 03, 008, arXiv:1607.02139 [astro-ph.C0].

[4] DES, SPT Collaboration, B. Soergel et al., "Detection of the kinematic Sunyaev-Zel'dovich effect with DES Year 1 and SPT," Mon. Not. Roy. Astron. Soc. 461 (2016) no. 3, 3172-3193, arXiv:1603.03904 [astro-ph.CO].

[5] Planck Collaboration, P. A. R. Ade et al., "Planck intermediate results. XXXVII. Evidence of unbound gas from the kinetic Sunyaev-Zeldovich effect," Astron. Astrophys. 586 (2016) A140, arXiv:1504.03339 [astro-ph.CO].

[6] CMB-S4 Collaboration, K. N. Abazajian et al., "CMB-S4 Science Book, First Edition," arXiv:1610.02743 [astro-ph.CO].

[7] R. A. Sunyaev and Ya. B. Zeldovich, "The Velocity of clusters of galaxies relative to the microwave background. The Possibility of its measurement," Mon. Not. Roy. Astron. Soc. 190 (1980) 413-420.

[8] M. Kamionkowski and A. Loeb, "Getting around cosmic variance," Phys. Rev. D56 (1997) 4511-4513 arXiv: astro-ph/9703118 [astro-ph].

[9] R. R. Caldwell and A. Stebbins, "A Test of the Copernican Principle," Phys. Rev. Lett. 100 (2008) 191302 arXiv:0711.3459 [astro-ph]

[10] P. Zhang and A. Stebbins, "Confirmation of the Copernican principle at Gpc radial scale and above from the kinetic Sunyaev Zel'dovich effect power spectrum," Phys. Rev. Lett. 107 (2011) 041301, arXiv:1009.3967 [astro-ph.CO].

[11] T. Clifton, C. Clarkson, and P. Bull, "The isotropic blackbody CMB as evidence for a homogeneous universe," Phys. Rev. Lett. 109 (2012) 051303, arXiv:1111.3794 [gr-qc].

[12] R. Maartens, "Is the Universe homogeneous?," Phil. Trans. Roy. Soc. Lond. A369 (2011) 5115-5137. arXiv: 1104.1300 [astro-ph.CO].

[13] J. P. Zibin and A. Moss, "Linear kinetic Sunyaev-Zel'dovich effect and void models for acceleration," Class. Quant. Grav. 28 (2011) 164005, arXiv:1105.0909 [astro-ph.C0].

[14] P. Bull, T. Clifton, and P. G. Ferreira, "The kSZ effect as a test of general radial inhomogeneity in LTB cosmology," Phys. Rev. D85 (2012) 024002, arXiv:1108.2222 [astro-ph.C0].

[15] C.-M. Yoo, K.-i. Nakao, and M. Sasaki, "CMB observations in LTB universes: Part II - the kSZ effect in an LTB universe," JCAP 1010 (2010) 011, arXiv:1008.0469 [astro-ph.C0].

[16] P. Zhang and M. C. Johnson, "Testing eternal inflation with the kinetic Sunyaev Zel'dovich effect," JCAP 1506 (2015) no. 06, 046, arXiv:1501.00511 [astro-ph.CO].

[17] A. Terrana, M.-J. Harris, and M. C. Johnson, "Analyzing the cosmic variance limit of remote dipole measurements of the cosmic microwave background using the large-scale kinetic Sunyaev Zel'dovich effect," JCAP 1702 (2017) no. 02, 040, arXiv:1610.06919 [astro-ph.C0].

[18] A.-S. Deutsch, E. Dimastrogiovanni, M. C. Johnson, M. Münchmeyer, and A. Terrana, "Reconstruction of the remote dipole and quadrupole fields from the kinetic Sunyaev Zel'dovich and polarized Sunyaev Zel'dovich effects," arXiv:1707.08129 [astro-ph.C0]. 
[19] S. Ho, S. Dedeo, and D. Spergel, "Finding the Missing Baryons Using CMB as a Backlight," arXiv:0903.2845 [astro-ph.CO].

[20] P. Zhang, "The dark flow induced small scale kinetic Sunyaev Zel'dovich effect," Mon. Not. Roy. Astron. Soc. 407 (2010) L36, arXiv:1004.0990 [astro-ph.CO].

[21] J. Shao, P. Zhang, W. Lin, Y. Jing, and J. Pan, "The kinetic SZ tomography with spectroscopic redshift surveys," Mon. Not. Roy. Astron. Soc. 413 (2011) 628-642, arXiv:1004.1301 [astro-ph.C0]

[22] T. Okamoto and W. Hu, "CMB lensing reconstruction on the full sky," Phys. Rev. D67 (2003) 083002 , arXiv:astro-ph/0301031 [astro-ph].

[23] C. Dvorkin and K. M. Smith, "Reconstructing Patchy Reionization from the Cosmic Microwave Background," Phys. Rev. D79 (2009) 043003, arXiv:0812.1566 [astro-ph]

[24] LSST Science, LSST Project Collaboration, P. A. Abell et al., "LSST Science Book, Version 2.0," arXiv:0912.0201 [astro-ph.IM]

[25] S. Dodelson, Modern Cosmology. Academic Press, Amsterdam, 2003. http://www.slac.stanford.edu/spires/find/books/www?cl=QB981:D62:2003.

[26] C. Howlett, M. Manera, and W. J. Percival, "L-PICOLA: A parallel code for fast dark matter simulation," Astron. Comput. 12 (2015) 109-126, arXiv:1506.03737 [astro-ph.C0].

[27] R. Scoccimarro, L. Hui, M. Manera, and K. C. Chan, "Large-scale Bias and Efficient Generation of Initial Conditions for Non-Local Primordial Non-Gaussianity," Phys. Rev. D85 (2012) 083002, arXiv: 1108.5512 [astro-ph.CO].

[28] S. Tassev, M. Zaldarriaga, and D. Eisenstein, "Solving Large Scale Structure in Ten Easy Steps with COLA," JCAP 1306 (2013) 036, arXiv:1301.0322 [astro-ph.CO].

[29] G. Tormen and E. Bertschinger, "Adding long wavelength modes to an n-body simulation," Astrophys. J. 472 (1996) 14, arXiv:astro-ph/9512131 [astro-ph].

[30] S. Cole, "Adding long-wavelength power to n-body simulations," Mon. Not. Roy. Astron. Soc. 286 (1997) 38 arXiv:astro-ph/9604046 [astro-ph].

[31] E. Bertschinger, "Multiscale Gaussian random fields for cosmological simulations," Astrophys. J. Suppl. 137 (2001) 1, arXiv:astro-ph/0103301 [astro-ph]

[32] R. Sgier, A. Réfrégier, A. Amara, and A. Nicola, "Fast Generation of Covariance Matrices for Weak Lensing," arXiv:1801.05745 [astro-ph.CO].

[33] R. Teyssier, S. Pires, S. Prunet, D. Aubert, C. Pichon, A. Amara, K. Benabed, S. Colombi, A. Refregier, and J.-L. Starck, "Full-Sky Weak Lensing Simulation with 70 Billion Particles," Astron. Astrophys. 497 (2009) 335, arXiv:0807.3651 [astro-ph].

[34] A. Kiessling, A. F. Heavens, and A. N. Taylor, "SUNGLASS: A new weak lensing simulation pipeline," Mon. Not. Roy. Astron. Soc. 414 (2011) 2235, arXiv:1011.1476 [astro-ph.C0].

[35] S. Flender, L. Bleem, H. Finkel, S. Habib, K. Heitmann, and G. Holder, "Simulations of the Pairwise Kinematic Sunyaev-Zel'dovich Signal," Astrophys. J. 823 (2016) no. 2, 98, arXiv:1511.02843 [astro-ph.C0]

[36] K. M. Gorski, E. Hivon, A. J. Banday, B. D. Wandelt, F. K. Hansen, M. Reinecke, and M. Bartelman, "HEALPix - A Framework for high resolution discretization, and fast analysis of data distributed on the sphere," Astrophys. J. 622 (2005) 759-771, arXiv:astro-ph/0409513 [astro-ph]

[37] J. Koda, C. Blake, T. Davis, C. Magoulas, C. M. Springob, M. Scrimgeour, A. Johnson, G. B. Poole, and L. Staveley-Smith, "Are peculiar velocity surveys competitive as a cosmological probe?," Mon. Not. Roy. Astron. Soc. 445 (2014) no. 4, 4267-4286, arXiv:1312.1022 [astro-ph.CO]

[38] N. E. Chisari and M. Zaldarriaga, "Connection between Newtonian simulations and general relativity," Phys. Rev. D83 (2011) 123505, arXiv:1101.3555 [astro-ph.C0]. [Erratum: Phys. Rev.D84,089901(2011)].

[39] C. Fidler, T. Tram, C. Rampf, R. Crittenden, K. Koyama, and D. Wands, "Relativistic Interpretation of Newtonian Simulations for Cosmic Structure Formation," JCAP 1609 (2016) no. 09, 031, arXiv:1606.05588 [astro-ph.CO].

[40] W. E. East, R. Wojtak, and T. Abel, "Comparing Fully General Relativistic and Newtonian Calculations of Structure Formation," Phys. Rev. D97 (2018) no. 4, 043509, arXiv:1711.06681 [astro-ph.C0]

[41] D. Blas, J. Lesgourgues, and T. Tram, "The Cosmic Linear Anisotropy Solving System (CLASS) II: Approximation schemes," JCAP 1107 (2011) 034, arXiv:1104.2933 [astro-ph.CO].

[42] V. Böhm, M. Schmittfull, and B. D. Sherwin, "Bias to CMB lensing measurements from the bispectrum of large-scale structure," Phys. Rev. D94 (2016) no. 4, 043519, arXiv:1605.01392 [astro-ph.C0].

[43] A. Lewis and A. Challinor, "Weak gravitational lensing of the cmb," Phys. Rept. 429 (2006) 1-65, arXiv:astro-ph/0601594 [astro-ph].

[44] H. Park, M. A. Alvarez, and J. R. Bond, "The Impact of Baryonic Physics on the Kinetic Sunyaev-Zel'dovich Effect," Astrophys. J. 853 (2018) no. 2, 121, arXiv: 1710.02792 [astro-ph.CO]. 